\title{
Robotic Cystectomy : Important considerations before commencing the procedure independently
}

\author{
Nikhil Vasdev, Ben Lamb, Tim Lane, Gregory Boustead, James M Adshead \\ Hertfordshire and South Bedfordshire Urological Robotic Centre, Department of Urology, Lister Hospital, Stevenage, UK
}

Received September 03, 2013; Accepted September 18, 2013; Published Online September 20, 2013

\section{Scientific Note}

A radical cystectomy ( $\mathrm{RC}$ ) with pelvic lymph node dissection (PLND) is the gold standard for the management of the appropriately selected patient with muscle invasive bladder cancer (MIBC) and non-muscle invasive bladder cancer (NMIBC)/carcinoma in situ (CIS) who fail appropriate intravesical therapy. In the last decade, Robotic Radical Cystectomy (RRC) is being performed in a large number of international Centre's with the published advantages of decreased blood loss, improved post-operative convalescence and earlier initiation of adjuvant therapy ${ }^{1}$ when compared to open cystectomy (OC). Current literature indicates that a RC is equivalent to $\mathrm{OC}$ from the oncological perspective. An OC is associated with high rates of morbidity (19-64\%) or mortality (6-11\%), although there is a wide variation in current literature. ${ }^{1-11}$ A RRC is perhaps just one modality in a raft of measures to try reducing mortality and morbidity of a cystectomy.

To the Robotic Urological Surgeon, a RRC comes with numerous specific challenges. Questions that arise at the time of commencing a RRC include the learning curve of the procedure, learning steps to enhances ones speed to perform the procedure efficiently and safely, level of lymphnode dissection, whether one should embark of performing an intracorpealileal conduit or neobladder formation and the cost of commencing a RRC service. The patient's postoperative management is the most important step to ensure that the post-operative complications are kept to a minimum using a multi-disciplinary team (MDT) approach.

Corresponding author: Nikhil Vasdev, FRCS (Urol); Hertfordshire and South Bedfordshire Urological Robotic Centre, Department of Urology, Lister Hospital, Stevenage, UK.

Email: nikhilvasdev@doctors.org.uk

Cite this article as:

Vasdev N, Lamb B, Lane T, Boustead G, Adshead J. Robotic Cystectomy : Important considerations before commencing the procedure independently. Int J Cancer Ther Oncol 2013;1(1):01017. DOI: $10.14319 /$ ijcto.0101.7
In current literature high volume centers with experienced surgeons have reported patient outcomes that are acceptable from the perspective of extended pelvic lymph node dissection, positive surgical margin rates and highlight that patients are not being compromised from the surgical perspective in undergoing a RC. ${ }^{2}$

The learning curve of a RRC is not as clearly defined in comparison to Robotic Radical Prostatectomy (RRP). Before commencing aRRC it is important to be proficient and familiar with robotic pelvic surgery. Most robotic surgeons are proficient in RRP before embarking on performing independent RRC. Hayn et al. ${ }^{3}$ have indicated that an acceptable level of proficiency to perform a RRC is established after the $30^{\text {th }}$ case by measuring post-operative parameters such as operative time, lymph node yield (LNY), estimated blood loss (EBL), and margin positivity. At our center we commenced performing RC after performing 150 Robotic RRP. We would strongly recommend that a robotic urological surgeon who is keen to commence Robotic RC should be proficient in robotic RRP and in performing an extended pelvic lymph node dissection (EPLND). A well-trained Robotic Team consisting of the lead experience console surgeon, experienced assistant, nursing staff and an experience anesthetist is essential for the commencement of a RRC program. The techniques that a team needs to develop to aid in improving intra-operative times including a fast docking/ undocking time, piggyback techniques for ports and, the use of different specimen retrieval bags, use of laparoscopic staplers and new intraoperative hemostatic agents.

Whilst performing an adequate RRC involves the removal of the bladder, the importance of performing an EPLND cannot be understated. There is no defined lymph node dissection template for a RRC and some centers now perform an EPLND before performing the robotic cystectomy during a RRP. ${ }^{4}$ The anatomical landmark that we recommend to be followed is up to the level of the aortic bifurcation or the Inferior mesenteric artery (IMA), lymph node of cloquet distally, genitofemoral nerve laterally and perivesical tissue laterally. Using this template the surgeon will be able to excise the external iliac, obturator, hyogastic and common 
iliac. Some authors ${ }^{5}$ recommend the excision of the presacrallymphnode as the excision of this lymph node group facilitates the transposition of the left ureter behind the sigmoid mesentery to aid in intracorporeal anastomosis.

With an evolution in robotic systems there has been an increase in the number of patients undergoing intracorporeal ileal conduit and neobladder formation. The excellent technical description of the procedure has led to the adoption of these techniques worldwide. ${ }^{5}$ We have recently published the results of our initial patients. ${ }^{6}$ Robotic surgeons must be proficient in the performing the cystectomy part of the RRC before embarking on either an intracorporeal ileal conduit or neobladders formation due the complications of keeping patients in the steep Trendelenburg position for prolonged periods, which include compartment syndrome, neurological complications, intraocular complications, and rhabdomyolysis. ${ }^{7,8}$ Recent evidence from high volume centres performing RRC and intracorporeal neobladders diversion confirm that initial results are comparable to contemporary open series with regards to complication rates, functional and oncological outcomes. ${ }^{9}$ We recommend that a surgeon should embark on performing an intracorporeal ileal conduit and neobladder formation only if the ablative part of $\mathrm{RRC}$ is less than 2.5 hours of total operative time. If the ablative times are longer we recommend performing a mini-laparotomy for excision of the cystectomy specimen and completing an extracorporeal ileal conduit or neobladders. ${ }^{6}$

The cost effectiveness of a RRC when compared to an open radical cystectomy (ORC) has a disadvantage of being more expensive due to the initial high purchase and maintenance contract cost, although when the indirect costs of complications and extended hospital stay with ORC are considered, RRC may be less expensive than the traditional open procedure. In order to accurately evaluate the cost effectiveness of RARC versus ORC, prospective randomized trials between the two surgical techniques with long-term oncologic efficacy are needed. ${ }^{10}$

In conclusion, a RRC with intracorporeal ileal conduit or neobladders formation is a complex robotic procedure that should only be undertaken by an experienced robotic pelvic oncological surgeon who is completely competent at performing a RRP + EPLND. A carefully mentored approach and a well-trained robotic team are the two key components to make the procedure a success.

\section{Competing interests}

The authors declare that they have no conflicts of interest. The authors alone are responsible for the content and writing of the paper.

\section{References}

1. Liss MA, Kader AK. Robotic-assisted laparoscopic radical cystectomy: history, techniques and outcomes. World J Urol $2013 ; 31: 489-9$.

2. Khan MS, Elhage O, Challacombeet al B. Long-term outcomes of robot-assisted radical cystectomy for bladder cancer. Eur Urol 2013; 64: 219-224.

3. Hayn MH, Hussain A, Mansour AM et al. The learning curve of robot-assisted radical cystectomy: results from the International Robotic Cystectomy Consortium. Eur Urol 2010; 58: 197-202.

4. Desai MM, Berger AK, BrandinaRRet al. Robotic and laparoscopic high extended pelvic lymph node dissection during radical cystectomy: technique and outcomes. Eur Urol 2012; 61: 350-5.

5. Pruthi RS, Nix J, McRackan D, et al. Robotic-assisted laparoscopic intracorporeal urinary diversion. Eur Urol 2010; 57: 1013-21.

6. Bishop C, Vasdev N, Boustead G, Adshead JM. Robotic IntracorpealIleal conduit formation - Initial experience from a single UK centre. Advances in Urology [Article in press].

7. Mattei A, Di Pierro GB, Rafeld V, Konrad C, Beutler J, Danuser H. Positioning injury, rhabdomyolysis, and serum creatine kinase-concentration course in patients undergoing robot-assisted radical prostatectomy and extended pelvic lymph node dissection. J Endourol 2013; 27: 45-5.

8. Pandey R, Garg R, Darlong V, Punj J, Chandralekha, Kumar A. Unpredicted neurological complications after robotic laparoscopic radical cystectomy and ileal conduit formation in steep trendelenburg position: two case reports. ActaAnaesthesiol Belg 2010; 61: 163-6.

9. Tyritzis SI, Hosseini A, Collins J, Nyberg T, Jonsson MN, Laurin O, Khazaeli D, Adding C, Schumacher M, Wiklund NP. Oncologic, Functional, and Complications Outcomes of Robotic assisted Radical Cystectomywith Totally Intracorporeal Neobladder Diversion. Eur Urol 2013 (Article in press).

10. Mmeje CO, Martin AD, Nunez-Nateras R, Parker AS, Thiel DD, Castle EP. Cost analysis of open radical cystectomy versus robot-assisted radical cystectomy. Curr Urol Rep 2013;14: 26-3.

11. Prentis JM, Trenell MI, Vasdev N, French R, Dines G, Thorpe A, Snowden CP. Impaired cardiopulmonary reserve in an elderly population is related to postoperative morbidity and length of hospital stay after radical cystectomy. BJU Int 2013; 112: E13-9. 